\title{
Using Visual Communication Elements in Technical Reports
}

Coralee $\mathrm{McNee}^{1}$, Aaron Kueck ${ }^{2}$ and Dave Christie ${ }^{2}$

1. Metallurgist, Brooktondale, New York, United States.

2. Curtiss-Wright Corporation, Ithaca Materials Research, Lansing, New York, United States.

Visual examination, fractography and photodocumentation are arguably the most important pieces of analysis when running a metallurgical failure investigation. Each of these analyses requires communicating complex systems visually. Therefore, visual communication concepts can prove to be a valuable tool. As failure analysts often work with clients only briefly, their ability to communicate complex ideas effectively to a diverse audience can increase the likelihood of their success. This paper highlights the following key requirements for effective visual communications.

Be observant. In failure analysis, it is important to not only observe the fracture surface but the entire part, and it is important to understand how the part interacts with other components or surrounding environments. Approach problems without a preconceived idea of what the solution may be; avoid overlooking details that don't fit the "standard" narrative.

Composition counts. Composition includes balance, proportion, repetition, unity, focal point, rhythm and variety. An easy way to create balance in an image is to use the rule of thirds for placing your focal point in an image. Composition applies to sets of images as well. When documenting a single feature across several different imaging techniques, it is often useful to use the same or similar magnification and orientation, implementing unity. Using several different imaging techniques to capture a single feature provides repetition. For example capturing backscatter electron images, secondary electron images, incident light images, and diffuse light images provides variety. Combining unity and repetition in this way provides variety in the documentation of that feature. For example, images in sequence allow the viewer distinguish between relevant differences without explicit explanation as seen in Figure 1.

Also, consider a report as a single composition. The information, images, data and presentation should all connect to one another in a way that keeps the interest of the reader. For example, the report should move from macroscale photos to micrographs and from components to systems. Good composition often goes unnoticed and when done properly you are not aware of its function.

Repetition and unity should be used to communicate to standard information i.e. scale bars or font sizes in presentations. An added benefit is that careful use of repetition and unity make subtle variety a powerful tool in drawing the viewer's attention. Adding an annotated image to a presentation or using an inset in a diagram can focus your viewer without using intrusive presentation artifices. Good report composition brings the reader along with the failure analyst as the problem is described and documented, analyzed, and finally dispositioned. 
Figure captions should be descriptive but succinct and guide the reader to the relevant features in the image. Avoid long-winded explanations when a suitable image conveys the idea visually. Annotations on images should provide cues guiding the reader to important features, but without crowding the image or obscuring surrounding features. Complicated annotations may require a pair of images, with and without additions, to provide the complete picture.

Engineers and scientists communicate increasingly complex systems and specialized science to increasingly diverse audiences. Visual communication can transcend language, cultural, and technical backgrounds when done well. It is no surprise that when someone really wants to understand how something works they say 'Show me.'

\section{References:}

[1] A Ruedinger, "Visual Communication" Art Institute Portland, OR Summer 2007. Lecture. [2] D A Lauer and S Pentak, "Design Basics" $6{ }^{\text {th }}$ ed. (Thomson Wadsworth, United States) p. 23.
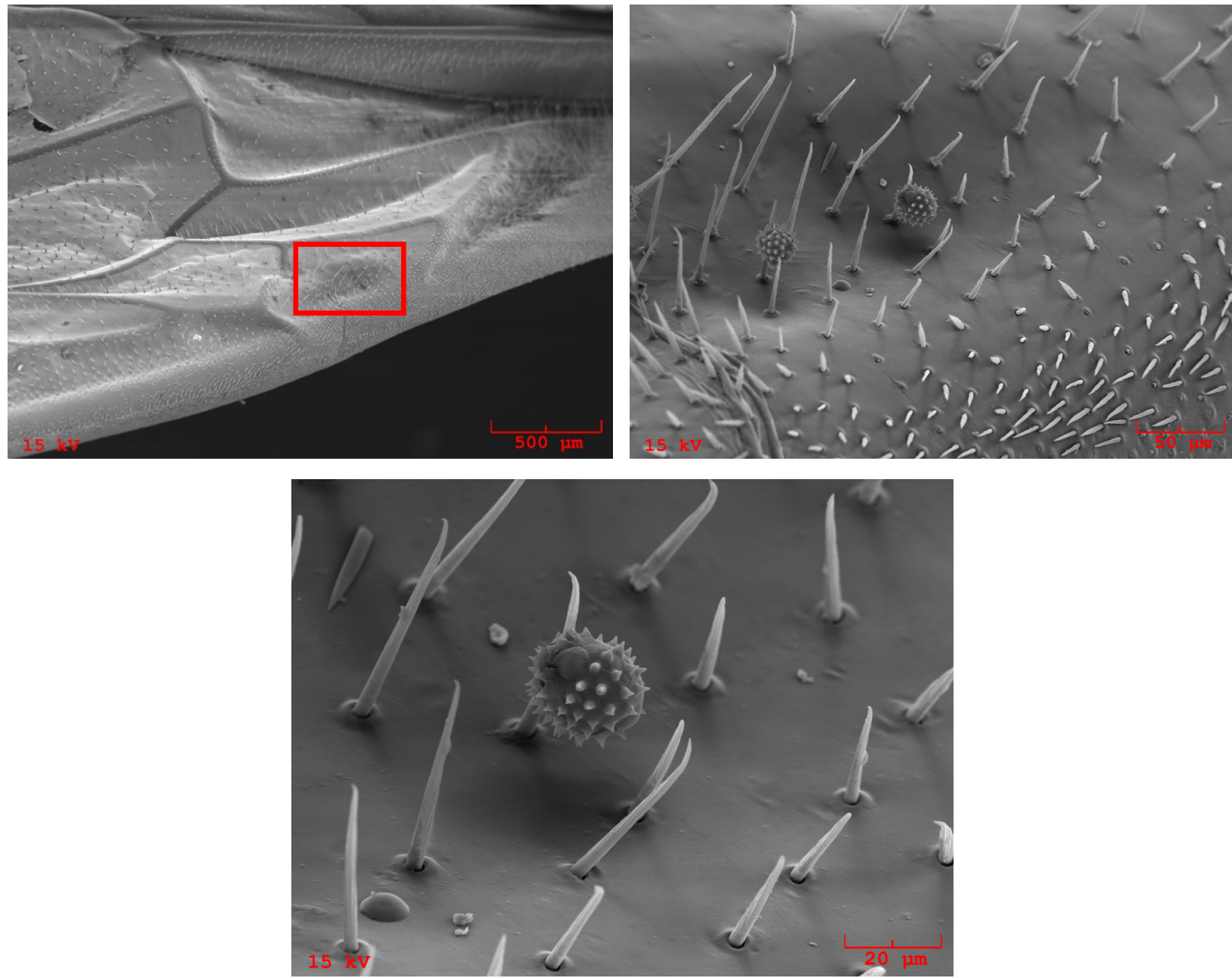

Figure 1. Using three images in sequence allows the viewer to locate the higher magnification image, while providing additional information about the subject. 